\title{
Employment of Wireless Sensor Networks for Full-Scale Ship Application
}

\author{
Bu-Geun Paik ${ }^{1}$, Seong-Rak Cho ${ }^{1}$, Beom-Jin Park ${ }^{1}$, Dongkon Lee ${ }^{1}$, \\ Jong-Hwui Yun ${ }^{2}$, and Byung-Dueg Bae ${ }^{2}$ \\ ${ }^{1}$ Maritime \& Ocean Engineering Research Institute, KORDI, 104 Shinseong St., \\ Yuseong-gu, Daejeon, 305-343, Korea \\ \{ppaik, scho, baracude, dklee\} @ moeri.re.kr \\ ${ }^{2}$ Korea Maritime University, 1 Dongsam-dong, Youngdo-ku, Busan, 606-791, Korea \\ \{jhyun, captcos\} @ hhu.ac.kr
}

\begin{abstract}
In this study, basic experiments regarding the wireless sensor network were conducted on a 3,000-ton-class training ship as the first step in applying the ubiquitous technology to a real ship. Various application fields of the technology in terms of the provision of safety and convenience on a ship would be extracted through these experiments. To efficiently adopt the ubiquitous technology for ship application, it is necessary to identify the stateof-the-art ubiquitous technology and to prepare countermeasures against the harsh environment of a ship. Especially, the characteristics of the wireless sensor network were investigated at the test bed ashore as well as on a real ship to figure out the complementary items before full-scale ship application.
\end{abstract}

Keywords: ubiquitous, ship, WSN (wireless sensor network), Zigbee, monitoring.

\section{Introduction}

During ship operation on the seas, it has different requirements according to its type and purpose. A commercial ship transports various kinds of cargo, such as containers, logs, ore, crude oil, and LNG (liquefied natural gas), to the destination port within a given time. Comfort and convenience are expected of a passenger ship. Military ships should have mobility and the capabilities that are necessary for it to be able to carry out its mission. Since a ship stays on the seas most of the time during its operation, greater convenience must be provided to its crew and passengers so that the efficiency and safety of its operation could be ensured.

A number of ship management technologies aimed at increasing the efficiency and safety of a ship have been developed and proposed by many researchers. The EU (European Community) has developed intelligent hull-monitoring systems to reduce the risk of structural failure, spills into the sea and damage to cargo, and to improve passenger safety and comfort [1]. Nguyen and Nelson [2] discussed an approach to integrating data collection and analysis of ship machinery for the assessment of the conditions and operational performance of the ship's equipment. Nielsen et al. [3] 
introduced the concept of the onboard management of wave-induced structural loads and ship motions through the measurement of relative wave motions, bending moment, and so on. Recently, Cho et al. [4] reported the ubiquitous sensor network technology for ship application and related basic experiment results.

The services for the crew and passengers of ships lag behind those for people ashore. On land, there are many opportunities to enjoy the ubiquitous technology and to have a convenient and safe life on account of it, such as through home automation or home network systems [5], tracking systems [6], security or safety systems, and health care systems [7]. For the crews and passengers of ships on the seas, however, it is not easy to acquire the benefits of ubiquitous technology because of several restrictions.

Most ship owners or shipping companies are not willing as of yet to provide comfort and convenience to their respective crews at the expense of higher operational costs, although the rate of automation and the level of safety have increased in the latest ships. Moreover, the aging of the currently operated ships could also make efficient operations difficult. For example, significant man hours are used in checking scattered spare parts on a ship and in manually recording a maintenance log. Much time and hard work is required in preserving and maintaining the ship's cargo because a few crew members are assigned to accomplish tasks related to the ship's cargo. The ubiquitous technology can be applied in various areas of ship operations so as to promote the latter's efficiency.

In this study, the wireless sensor network was employed as a ubiquitous technology on a real ship, and the ship's environment for such technology was investigated. The Zigbee platform was chosen for the sensor network field of the WSN system. In addition, several problems regarding real-ship application were identified, and basic experiments about the characteristics of WSN were conducted at the test bed ashore to provide reference data for the appropriate design of the WSN for a real ship. Especially, practical items concerning the communication depth of WSN and power consumption were discussed for the WSN design in a real ship. Through the WSN experiments in the main-engine room of the real ship, the increase of the communication depth was found to be necessary. The power consumption tests were carried out in the test bed and expected to provide a reference data for node deployments in a real ship.

\section{Characteristics of Ships and of the WSN Technologies}

A ship operates in a very harsh environment. Many steel plates are cut or welded together to make a block, and then a ship is constructed by putting many of these blocks together. This means that a ship is a big structure made mainly of steel. Besides this, the main engine of the ship transfers significant power to the propeller through a long shaft, for the propulsion of the ship. As the deckhouse, a superstructure on the upper deck of a ship, is located above the main-engine room, the crew members inside the deckhouse suffer from the vibration and noises induced by the propeller or main engine. Most of all, the ship motions, such as pitching, rolling, and heaving, can cause considerable inconvenience to the crew members and passengers according to the sea conditions. If the ubiquitous technology would be applied in ships, this kind of operational environment should be taken account of. 
The most well-known ubiquitous technology is the RFID tag. The RFID tag, which has a specific identification code, is attached to an object, and various services (e.g., location finding [8], remote control, management, and communications between objects) are made possible by it with the use of radio waves, which produce an awareness of the surrounding environment. The price of RFID tags using the ultrahigh frequency of $900 \mathrm{MHz}$ or the microwave of $2.4 \mathrm{GHz}$ has recently been lowered, and the price of RFID readers is going down as well. Although the RFID tag may ensure the safety and security of a ship's crew members and passengers (those carrying an RFID tag), it is very difficult to reliably recognize an RFID tag carried by a person who is moving about on the ship because of the essential characteristics of high frequency wave.

Another ubiquitous technology is WSN (wireless sensor network), which has been receiving considerable attention along with RFID. The WSN has a structure where several sensor networks are connected to an external network through a gateway. The sensor nodes send data to a sink node nearby, where the data from each node are accumulated before being transmitted to a gateway. The data transferred from the gateway can also be transmitted using satellite. The WSN technology has achieved significant growth because of the ad-hoc network technology and the routing protocol standard. Especially, wireless communication technologies such as WLAN (wireless LAN), Zigbee, and Bluetooth came into the spotlight in terms of the construction of efficient wireless networks. In other words, wireless communication has some problems to be solved for the successful ship application, such as the fact that it makes use of multipass and a radio wave screen due to the steel structure in a ship. As these problems can reduce the reliability of data transmission or recognition, it is necessary to prepare backup plans such as variations in network topologies and the addition of repeaters or relay antennas.

\section{Full-Scale Ship Tests Using the WSN}

\subsection{Data Delivery Ratio for Zigbee}

The particulars of the ship that was chosen for this study are shown in Table 1 . Hannara, a 3,000-ton-class training ship, has relatively many cabins because it was designed to be sailed to train many students. Fig. 1 shows photo of the ship Hannara. The deckhouse of Hannara consists of the following, arranged vertically (from the bottom to the top): the bottom deck, which houses the main-engine room; the second deck, for the accommodations of students; the shelter deck, for the accommodations of the crew, the boat deck, for the accommodations of faculty members; the training deck, for navigation training; and the bridge deck, which is used as a wheel house.

The preliminary WSN tests were conducted when the ship was moored to the port and when all the equipments in the main-engine room, except for the electric generator, were not being operated. The wireless communication tests were done in one cabin at the shelter deck, which is the middle deck of the ship. The door of the cabin is shown in Fig. 2. It was made of steel but its surface was covered with smooth materials. Data transmission tests were carried out using two ZPAs (Zigbee Protocol Analyzers), one sending 100 10-byte packets and the other receiving those packets. 
The RF (radio frequency) strength, indicating the RSSI(received signal strength indication) as well as the number of packets, was measured to evaluate the transmission quality. We performed the measurements 10 times at each case. When the sending ZPA (located within the cabin) and the receiving ZPA (located outside the cabin) were $1 \mathrm{~m}$ away from the door, a 98 100\% delivery ratio (= received packet number/sent packet number) and an RSSI of -27 -30 dBm were found, which are not bad for wireless communication. Here, $\mathrm{dBm}$ is an abbreviation for the power ratio in decibel $(\mathrm{dB})$ of the measured power referenced to one milliwatt $(\mathrm{mW})$. Zero $\mathrm{dBm}$ equals one milliwatt. The delivery ratio and RSSI were $76 \%$ and $-37 \mathrm{dBm}$, respectively, when the receiving ZPA was at an $8 \mathrm{~m}$ distance from the cabin door. Although the cabin door was closed, wireless communication was found to be possible when a sensor node is arranged near the door because the cabin door has a ventilation window at its lower part as well as a small gap between the wall and the door itself, as shown in Fig. 2.

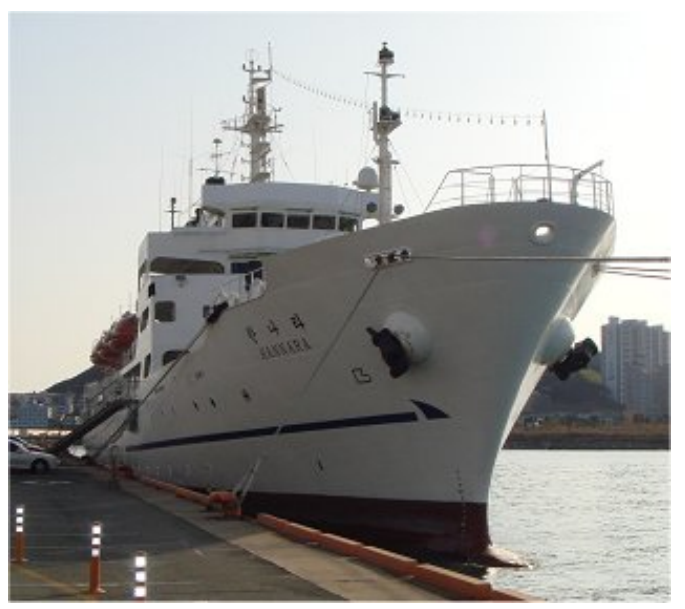

Fig. 1. Hannara for full-scale ship tests

The tests regarding delivery ratio were also conducted in the corridor fronting the cabin, with a width of $1.5 \mathrm{~m}$ and a length of about $40 \mathrm{~m}$. The sending ZPA was at the fixed location near one end of the corridor, and the receiving ZPA was moved to the other end of the corridor, $5 \mathrm{~m}$ away from the sending ZPA. The test results regarding the averaged delivery ratio and RF strength are shown in Table 2, and a photo of the corridor is shown in Fig. 3.

Table 2 shows that the wireless communication network will be on if two sensor nodes are within the line of sight, even at a distance of $40 \mathrm{~m}$. In this experiment, as each ZPA was positioned $1.5 \mathrm{~m}$ from the bottom, interference from crew members or passengers passing through the corridor is unavoidable. If the basic data about the possible interference from a person passing through the corridor would be prepared in the future, the optimized location of the sensor node or router without any interference would be found, and this could be used in designing the WSN for a real ship. 


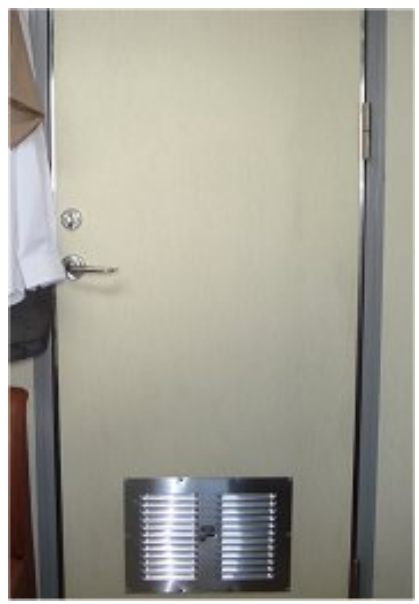

Fig. 2. Door of the cabin

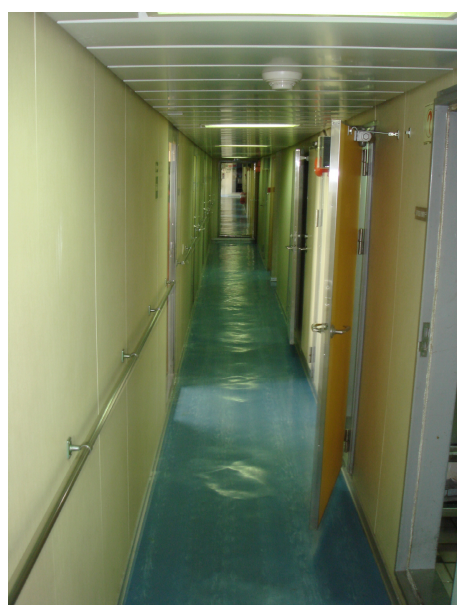

Fig. 3. Corridor in the shelter deck

The next stage was to measure the data delivery ratio at the stairs and between decks. The stairs from the shelter deck to the boat deck had a height of about $3 \mathrm{~m}$ and had steel walls with a thickness of over $15 \mathrm{~mm}$, providing a sort of shielding space from the microwave. The entrance door to the stairs at the shelter deck was opened, and the sending ZPA was positioned at the upper corner of the entrance. The receiving $\mathrm{ZPA}$, on the other hand, was positioned at the top of the stairs. The transmission ratio was $100 \%$, and the RSSI value was $-10 \mathrm{dBm}$, indicating very good communication. When the receiving ZPA at the boat deck was moved to different locations to remove it from the line of sight, the delivery ratio and RSSI value were $100 \%$ and $-14 \mathrm{dBm}$, respectively, at a distance of $2 \mathrm{~m}$ from the boat deck entrance. Even when the receiving ZPA at the boat deck was located just above the sending ZPA at the shelter deck, the delivery ratio and RSSI value were $98 \%$ and $-21 \mathrm{dBm}$, respectively

Table 1. Particulars of the ship Hannara

\begin{tabular}{|l|l|}
\hline Length (m) & 102.7 \\
\hline Width (m) & 14.5 \\
\hline Height (m) & 7 \\
\hline Tonnage (ton) & 3640 \\
\hline Speed (knot) & 15 (max. 17) \\
\hline Main Engine & $4,000 \mathrm{HP}$ diesel engine \\
\hline
\end{tabular}

The entrance door to the boat deck was always open, but the entrance door to the second deck, below the shelter deck, was made of steel and did not have any ventilation window. A delivery ratio of $63 \%$ and an RSSI of $-46 \mathrm{dBm}$ were obtained 
Table 2. Wireless Communication Tests for the Long Corridor in the Shelter Deck

\begin{tabular}{|c|c|c|}
\hline Distance (m) & Averaged delivery Ratio (\%) & Averaged RF Strength (dBm) \\
\hline 5 & 98 & -13 \\
\hline 10 & 100 & -12 \\
\hline 15 & 94 & -20 \\
\hline 20 & 100 & -17 \\
\hline 25 & 100 & -15 \\
\hline 30 & 98 & -27 \\
\hline 35 & 100 & -23 \\
\hline 40 & 100 & -25 \\
\hline
\end{tabular}

when the receiving ZPA was beyond the entrance door to the second deck, and when the steel door was closed. From these experiments, the wireless communication network was found to be on when the steel door of the entrance to each deck was open. In addition, the closing of the entrance door to each deck would seriously reduce the quality of the wireless communication through the stairs even if there was a small gap between the entrance door and the side walls. Therefore, the plans should be established in such a way as to ensure that the wireless communication would not be interrupted, by using some bypass antennas or replacing the wireless network with the wired network at the troubled region.

\subsection{WSN Tests in Main Engine-Room}

The basic WSN tests were conducted in the main-engine room, which had several equipments and was considered very important in the monitoring of the ship's environmental conditions. The main engine was stopped, but the electric generator was operated. Fig. 4 shows the main-engine room viewed from the main-engine control room, and the laptop computer and sink node were placed on the control console. Since various equipments such as pumps or filters, as well as the main engine, were arranged in a complex way inside the main-engine room, it was very important to properly employ the routers and sensor nodes. In the basic experiments, since there were no advance reference data about the delivery ratio or RSSI according to the microwave diffraction, the routers and sensor nodes were employed at places on the line of sight. A schematic diagram of the sensor network field is shown in Fig. 5. The sink node, indicating the gateway, was placed at the top of the console, whose height was $1.7 \mathrm{~m}$, inside the control room. Router 1 was placed on the top of an equipment that was $6 \mathrm{~m}$ away from the sink node and that had a height of $1.7 \mathrm{~m}$. Router 2 was placed on the top of a cylindrical steel structure that was $7 \mathrm{~m}$ away from router 1 and that was near the stairs leading to the second deck. Router 3 was placed at the entrance door to the second deck, and was about $4 \mathrm{~m}$ away from router 2 . Finally, the sensor nodes were positioned at the appropriate places based on the communication depth, and they communicated with each other from the main-engine 
control room to the utility room of the 2 nd deck by hopping. Sensor nodes 1 and 3 measured the temperature and humidity simultaneously. The intensity of illumination was measured by sensor nodes 2 and 4 . Router 2 had sensor nodes 1 and 2 as its children, while router 3 had sensor nodes 3 and 4 as its children. The communication depths of routers 1 and 2 were 1 and 2, respectively. The child of each router had a depth one step smaller than the router's depth. The empty space in the second deck pertains to the path of the large exhaust pipes for the main engine. After the operation of the WSN, it was confirmed that the wireless communication worked well and that it could be useful for the monitoring of equipment or for fire prevention.

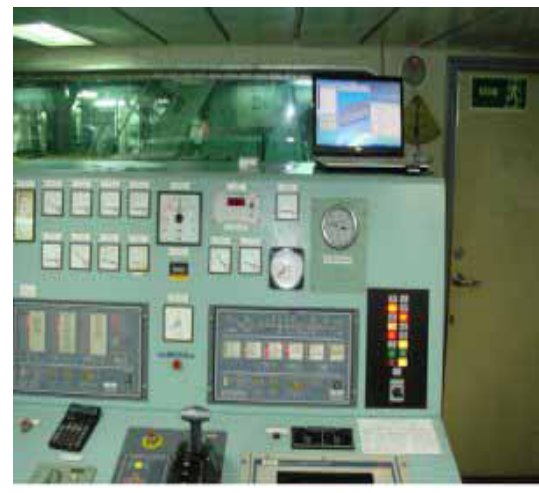

Fig. 4. Control room for main-engine

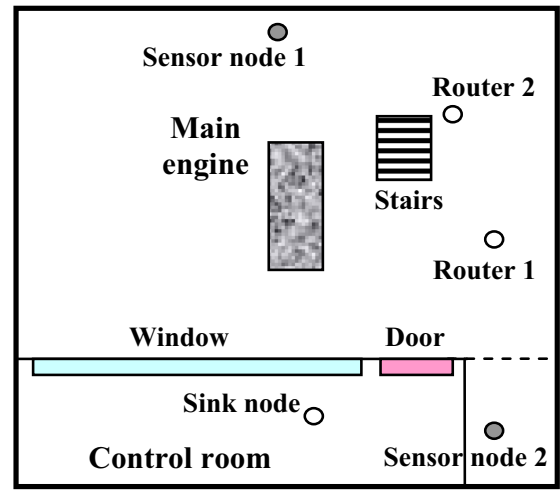

Fig. 5. Employment of WSN at the bottom deck (top view)

Several problems were found, although the basic experiments on the WSN that were conducted on a real ship were generally successful. The first problem was the communication depth of the WSN. Router 3 could allow its children to have only a fourth depth because the total depth of this WSN was four. Although the ship Hannara has a considerably small main-engine room, the monitoring of both the bottom and second decks was done partially due to the restricted communication depth of the WSN. In other words, the equipment and environment at the right side of the main-engine room can be monitored, but the monitoring of the equipment and environment at the left side is nearly impossible. In the second deck, the worst situation occurred from the viewpoint of the monitoring of equipment and environment. Therefore, it would be better to monitor only one deck in the case of the WSN with a small depth. To monitor two decks simultaneously, it is necessary to increase the depth of the WSN.

The second problem was related to the battery consumption of the sensor nodes. As most of the companies that are producing WSN modules design and manufacture these through approximate estimation, without accurate information regarding battery consumption, useful reference data are certainly necessary. There are some parts of the monitoring process that require a short sensing period, but the other parts may require a rather long sensing period. Power consumption tests were found to be necessary in terms of the sensing period. Especially, as the use of gas sensors for fire prevention will require significant power consumption, it would be desirable to adopt a regular power service. 
The third problem that was found concerns microwave diffraction. In the present study, the routers and sensor nodes were placed within the line of sight because there were no reference data about the diffraction. This, however, is not efficient for the WSN design because the communication depth can be consumed in vain to avoid obstacles within the line of sight. The basic data related to microwave diffraction are necessary for the WSN design together with the consideration of multipath effect in a ship.

The last problem that was found concerns placement. Although the main engine was not actually operated, there were parts of the main engine that had a high temperature at the bottom deck. As the positioning of the routers and sensor nodes near the parts of the main engine with a high temperature would result in damages on the WSN modules, the placement of the modules should be carefully considered. In addition, high vibration and noises as well as high-temperature parts should be treated with caution when the main engine is being operated.

\section{Investigation of the WSN Characteristics at the Test Bed}

\subsection{Battery Consumption Tests}

It is necessary to investigate the characteristics of the sensor node for the appropriate application of it to a real ship. Although the sensor node of the present WSN needs low power instead of regular power, the lowest battery limit for its normal operation, and the life of the battery, should be checked in advance. Especially, if the crew must frequently replace each battery with a new one during a long-term voyage, which is expected to last for over 10 days, the crew would be annoyed and might no longer want to use the WSN system. Thus, basic data concerning the time of a battery replacement, as well as the battery consumption, will be helpful for the application of the WSN to a ship.

In the case of the use of a sensor node encouraged by Zigbee alliance, the request from the sink or coordinator can change the sleep mode into the wake mode, and vice versa. However, as this method requires that each sensor node be ready to continuously recognize requests from the sink, the sensor node does not completely realize low power consumption. The sensor nodes that were used in this study have a micro clock outside their RF and MCU chips, which would allow them to synchronize the operation modes and to realize low power consumption because only the micro clock would be awakened, and the other chips would continue being in the sleep mode. In spite of the sensor nodes' realization of low power consumption, the instability of the WSN system due to its fast power consumption should be considered at the operation mode with a short sleeping period. In this study, battery tests related to the sensor nodes were conducted at the test bed on land.

To investigate the power consumption rate of each battery, the remaining voltage in the battery was measured for the temperature and gas sensor nodes with an $8 \mathrm{sec}$ sleeping period. The voltage of the battery was initially $3.4 \mathrm{~V}$, and the voltage value was measured continuously for about $100 \mathrm{hr}$ from 2 p.m. The lowest voltage limits of the temperature and gas sensor nodes were $2.73 \mathrm{~V}$ and $2.23 \mathrm{~V}$, respectively. The communication was interrupted because the RF module stopped operating when the 
remaining voltage in the battery became lower than the minimum voltage required. Fig. 6 shows the remaining voltage and temperature measured according to the time variation. The power of the battery was consumed independently of the temperature value, and the remaining voltage in the battery had a smooth and stable time history. In addition, since the temperature sensor node sensitively detected the ambient temperature, it can be said that the temperature environment can be controlled in the future by monitoring the temperature as part of context awareness. In the case of the gas sensor node, it excessively consumed the power of the battery from the beginning of the gas monitoring. The remaining voltage started from about $2.9 \mathrm{~V}$ and reached the minimum voltage required within $40 \mathrm{hr}$, as shown in Fig. 7. The sleeping time of 8 sec. was too short for the gas sensor node to be appropriately operated, and a time period of over a minute would be required for a much longer operation. Consequently, it is inappropriate for the gas sensor node to have the ability of low power consumption for the monitoring of natural gas, methane, butane, and other gases to prevent an explosion or fire in a real ship. The gas sensor node with regular power, indicated by a power adaptor, would instead be desirable for the real application of the WSN system.

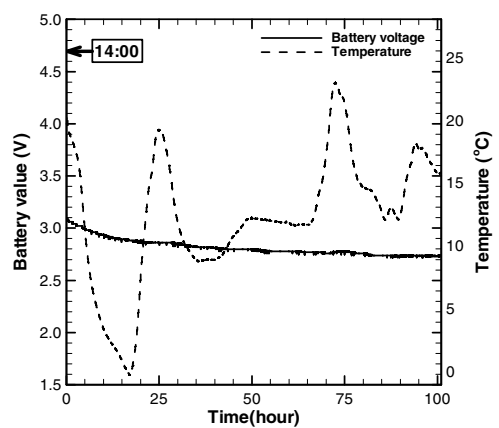

Fig. 6. Temperature sensor node

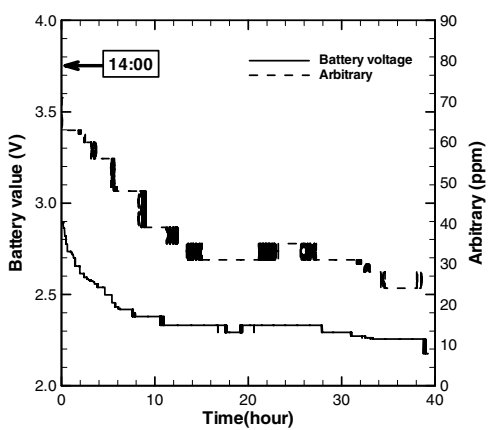

Fig. 7. Gas sensor node

When the sleeping time of the temperature sensor node became $64 \mathrm{sec}$, the remaining voltage was $2.88 \mathrm{~V}$ after about $205 \mathrm{hr}$. Based on its relation with the voltage consumption data of the 8 -sec period, the life of the battery was predicted to be about 15 days. The power consumption characteristics of the battery should be understood beforehand because the ship owner or crew would be afraid to use the WSN system with sensor nodes whose battery has a short life.

\section{Conclusion}

In the present study, a monitoring system was introduced for the abnormal operation of equipment and for fire prevention in dangerous regions, which are important application fields of the WSN technology. Several realistic problems were found from the basic WSN tests that were conducted on a real ship. The regular wireless communication is generally possible within a ship; however, the reference data 
regarding the communication depth, the power consumption rate of the battery in a sensor node, and the placement of the RF modules should be obtained more for the WSN design of a full-scale ship. In addition, the enduring time of each sensor node should be investigated carefully considering the multipath effects to provide a real ship with a useful WSN system besides the battery consumption tests at the test bed on land.

Acknowledgments. This research was accomplished with support from the inherent research project entitled "Development of smart operation technologies for exploration fleets based on the ubiquitous concept (PE0116A)."

\section{References}

1. MARINTEK., Intelligent hull-monitoring systems for the reduced risk of structural failure, spill into the sea, and damage to cargo, and for improved passenger safety and comfort (HULLMON+). G3RD-CT-2000-00329, EU Framework Programme (2000)

2. Nguyen, T.V., Nelson, H.W.: A systems approach to machinery condition monitoring and diagnosis. In: NDIA: Proceedings of the 4th Annual Systems Engineering Conference (2001)

3. Nielsen, J.K., Pedersen, N.H., Michelsen, J., Nielsen, U.D., Baatrup, J., Jensen, J.J., Petersen, E.S.: Sea sense: real-time onboard decision support. Annual Report, Force Technology (2006)

4. Cho, S.R., Lee, D.K., Paik, B.G., Yoo, J.H., Park, Y.H., Park, B.J.: A study on USN technologies for ships. In: Proceedings of Ubiquitous Intelligence and Computing, July 1113, 2007, Hong Kong, China (2007)

5. Hakem, N., Misson, M.: Study of the throughput of the wireless home automation network using the encapsulation of two medium-access methods. In: Proceedings of Communication Systems and Networks, September 9-12, 2002, Spain (2002)

6. Oppermann, L., Broll, G., Capra, M., Benford, S.: Extending authorizing tools for locationaware applications with an infrastructure visualization layer. In: Proceedings of Ubiquitous Computing, September 17-21, 2006, Orange County, CA, USA (2006)

7. Hodges, S., Williams, L., Berry, E., Izadi, S., Srinivasan, J., Butler, A., Smyth, G., Kapur, N., Wood, K.: SenseCam: a retrospective memory aid. In: Proceedings of Ubiquitous Computing, September 17-21, 2006, Orange County, CA, USA (2006)

8. Yun, K.H., Choi, S.W., Kim, D.J.: A robust location tracking system using the ubiquitous RFID wireless network. In: Proceedings of Ubiquitous Intelligence and Computing, Wuhan, China, September 3-6, 2006 (2006) 